\title{
ТЕОРЕТИЧЕСКИЕ ИССЛЕДОВАНИЯ КРЕСТООБРАЗНОГО ГЛУБИННОГО УПЛОТНИТЕЛЯ БЕТОННЫХ СМЕСЕЙ
}

\section{А. Г. Маслов, Жанар Батсайхан}

Кременчугский национальный университет имени Михаила Остроградского

ул. Первомайская, 20, 39600, м. Кременчуг, Украина. E-mail: kmto.43@gmail.com

Приведена расчетная схема крестообразного глубинного уплотнителя, взаимодействующего с бетонной средой, физико-механические свойства которой представлены в виде упругих, диссипативных и инерционных сил. Составлена система уравнений, описывающая прямолинейные колебания центра тяжести исследуемой динамической системы в горизонтальной плоскости во взаимно-перпендикулярных направлениях, а также крутильные колебания относительно координатных осей, проходящих через центр тяжести крестообразного глубинного уплотнителя. Установлена закономерность движения рабочих поверхностей крестообразного уплотняющего наконечника в горизонтальной плоскости во взаимно-перпендикулярных направлениях и вызывающего в бетонной смеси переменное амплитудно-частотное вибрационное воздействие, которое обеспечивает в уплотняемой среде нормальное напряженно-деформированное состояние с изменяющимися параметрами в горизонтальной плоскости, являющееся определяющим в разрушении структурных связей, переводу бетонной смеси в тиксотропное состояние. Полученные результаты исследований позволяют обосновать рациональные параметры и режимы вибрационного воздействия крестообразного глубинного уплотнителя на бетон различной консистенции.

Ключевые слова: крестообразный глубинный уплотнитель, бетонная смесь, вибрационное уплотнение.

\section{ТЕОРЕТИЧНІ ДОСЛІДЖЕННЯ}

\section{О. Г.Маслов, Жанар Батсайхан}

Кременчуцький національний університет імені Михайла Остроградського

вул. Першотравнева, 20, 39600, м. Кременчук, Україна. E-mail: kmto.43@gmail.com

Наведено розрахункову схему хрестоподібного глибинного ущільнювача, що взаємодіє з бетонним середовищем, фізико-механічні властивості якого представлені у вигляді пружних, дисипативних та інерційних сил. При цьому хрестоподібний глибинний ущільнювач складається з занурюваного в бетонну суміш ущільнюючого наконечника, виконаного у вигляді сполучених хрестовим чином вертикальних плит. На верхній частині ущільнюючого хрестоподібного наконечника за допомогою опорного кільця змонтовано віброзбуджувач кругових коливань. Складена система рівнянь, що описує прямолінійні коливання центру тяжіння досліджуваної динамічної системи в горизонтальній площині у взаємно-перпендикулярних напрямках, а також крутильні коливання відносно координатних осей, що проходять через центр тяжіння хрестоподібного глибинного ущільнювача. Визначені моменти інерції коливної динамічної системи з урахуванням фізико-механічних характеристик ущільнюваного середовища, знайдені коефіцієнти жорсткості кручення і непружного опору бетонної суміші. Встановлено закономірність руху робочих поверхонь хрестоподібного ущільнюючого наконечника в горизонтальній площині у взаємно-перпендикулярних напрямках, що викликає в бетонній суміші змінну амплітудночастотну вібраційну дії, яка забезпечує в ущільнюваному середовище нормальне напружено-деформоване становище із змінними параметрами в горизонтальній площині, що $€$ визначальним у руйнуванні структурних зв'язків, переведення бетонної суміші в тиксотропное стан. Отримані результати досліджень дозволяють: обгрунтувати раціональні параметри хрестоподібного глибинного ущільнювача, що здійснює просторові коливання $\mathrm{i}$ викликає в бетонній суміші змінні амплітудно-частотні деформації, що сприяють ефективному ущільненню; знайти раціональні режими вібраційного впливу на бетонні суміші різної консистенції. Пропонований хрестоподібний глибинний ущільнювач може знайти широке застосування в практиці будівництва в монолітному будівництві, при зведенні залізобетонних виробів, конструкцій і фундаментів, при виготовленні бетонних блоків, несучих колон, плит перекриття, сходів і т. п.

Ключові слова: хрестоподібний глибинний ущільнювач, бетонна суміш, вібраційне ущільнення.

АКТУАЛЬНОСТЬ РАБОТЫ. В практике современного строительства широко используют для уплотнения бетонных смесей глубинные вибраторы [1-4]. Их применяют в монолитном домостроении, при заливке железобетонных изделий, конструкций и фундаментов, при изготовлении бетонных блоков, несущих колонн, плит перекрытия, лестниц и т.д. [5, 6]. С целью повышения производительности, снижения энергоемкости, повышения надежности и упрощения конструкции был разработан крестообразный глубинный уплотнитель [7], предназначенный для уплотнения бетонных смесей различной консистенции. Для обеспечения эффективной работы предлагаемого уплотнителя необходимо точно определить его основные параметры и режимы работы в зависимости от физико-механических характеристик уплотняемой среды. Поэтому проведение исследований, обеспечивающих создание высокопроизводительного и высокотехнологичного глубинного уплотнителя простой конструкции, имеющего высокую надежность и обеспечивающего уплотнение бетонных смесей различной консистенции, является актуальной задачей.

Цель работы - определение теоретическим путем рациональных параметров крестообразного глубинного вибрационного уплотнителя бетонных смесей различной консистенции. 
МАТЕРИАЛ И РЕЗУЛЬТАТЫ ИССЛЕДОВАНИЙ. Для определения закона движения вертикальных плит, непосредственно взаимодействующих с бетонной смесью в рабочем режиме, рассмотрим расчетную модель динамической системы «крестовый глубинный уплотнитель - бетонная среда» (рис. 1). Перемещения представленной динамической системы будем рассматривать, как в направлении координатной оси $X$, так и в направлении координатной оси $Y$, проходящих через центр тяжести колеблющейся динамической системы. Под действием горизонтальной возмущающей силы, развиваемой вибровозбудителем колебаний, вертикаль- ные плиты, взаимодействующие с бетонной смесью, приобретают сложные движения: а) линейные перемещения в направлении координатной оси $X$ и крутильные колебания относительно координатной оси $Y$, проходящие через центр тяжести $C$ колеблющейся системы; б) линейные перемещения в направлении координатной оси $Y$ и крутильные колебания относительно координатной оси $X$. При этом вертикальная плита 2 перемещается в направлении координатной оси $X$, а вертикальная плита 1 - в направлении координатной оси $Y$.

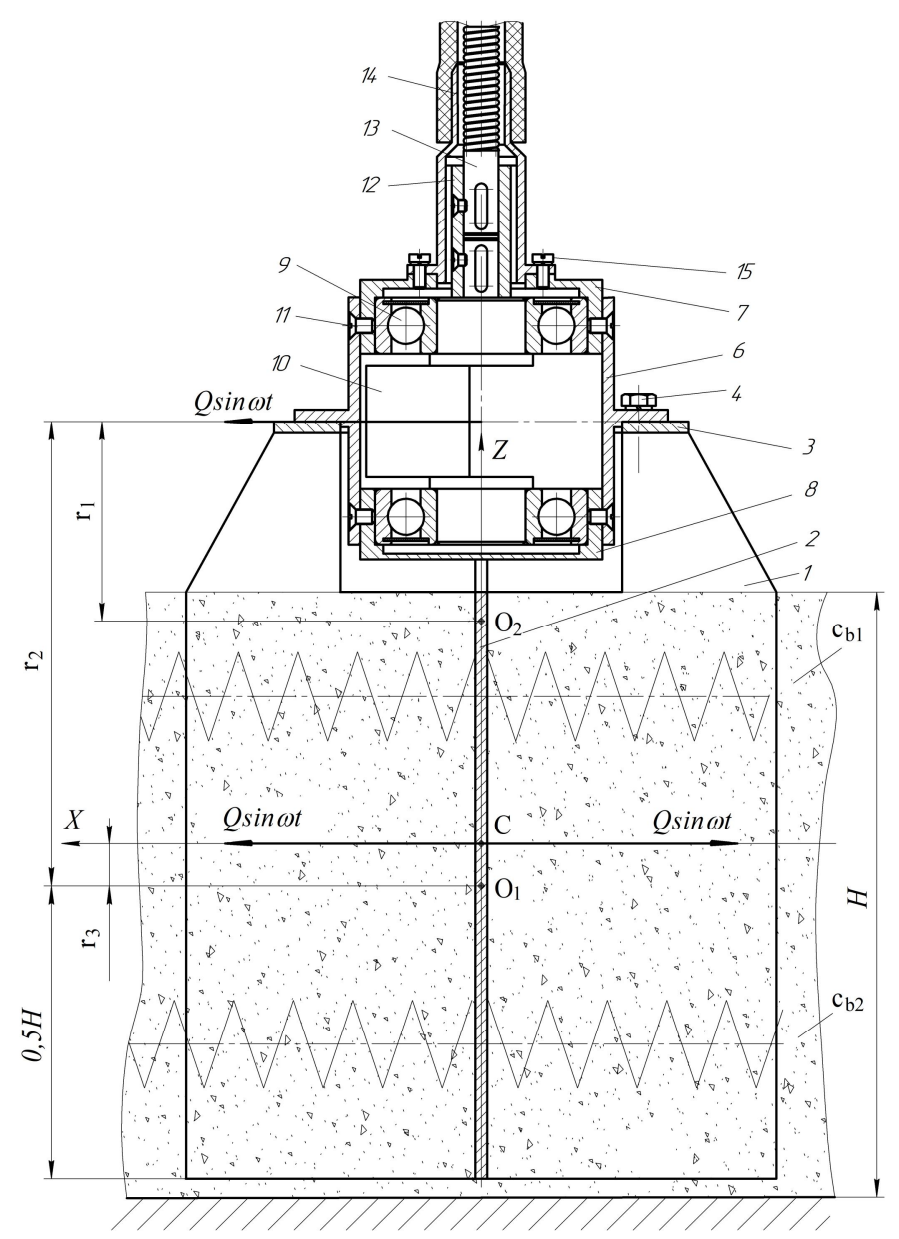

Рисунок 1 - Расчетная схема крестообразного глубинного виброуплотнителя: 1, 2 - вертикальные плиты; 3 - опорное кольцо; 4 - резьбовое соединение; 5 - вибровозбудитель колебаний; 6 - тонкостенный корпус; 7, 8 - проходная и глухая подшипниковые опоры; 9 - подшипники; 10 - дебаланс; 11 - винты;

12 - втулочная муфта; 13 - гибкий вал; 14 - кожух; 15 - винты

Смещение центра тяжести $C$ колеблющейся системы относительно центра тяжести бетонной смеси $O_{1}$ по вертикали определим из следующей зависимости:

$$
r_{3}=\frac{m\left(r_{2}-r_{1}\right)}{m+m_{b}},
$$

где $m$ - масса глубинного уплотнителя; $r_{1}$ - расстояние от центра тяжести вибровозбудителя колебаний до центра тяжести крестообразного глубинного уплотнителя; $r_{2}$ - расстояние от центра тяжести вибровозбудителя колебаний до центра тяжести приведенной массы бетонной смеси; $m_{b}$ - приведенная масса бетонной смеси, определяемая выражениями, приведенными в работах [8] или [9] в зависимости от способа вибрирования бетонной смеси: в полупространстве [8] или в форме [9].

Движение вибрационного наконечника крестообразного глубинного уплотнителя, контактирующего своими вертикальными плитами с бетонной смесью, можно описать следующей системой уравнений:

- прямолинейные перемещения в горизонтальной плоскости в направлении координатных осей $X$ и $Y$ : 


$$
\begin{gathered}
m \frac{d^{2} x}{d t^{2}}+m_{b}\left(\frac{d^{2} x}{d t^{2}}+f \frac{d^{2} y}{d t^{2}}\right)+b_{b}\left(\frac{d x}{d t}+f \frac{d y}{d t}\right)+ \\
+c_{b}(x+f y)=Q \sin \omega t \\
m \frac{d^{2} y}{d t^{2}}+m_{b}\left(\frac{d^{2} y}{d t^{2}}+f \frac{d^{2} x}{d t^{2}}\right)+b_{b}\left(\frac{d y}{d t}+f \frac{d x}{d t}\right)+ \\
+c_{b}(y+f x)=Q \cos \omega t
\end{gathered}
$$

- угловые перемещения относительно координатных осей $Y$ и $X$ :

$$
\begin{aligned}
& J \frac{d^{2} \psi}{d t^{2}}+J_{b}\left(\frac{d^{2} \psi}{d t^{2}}+f \frac{d^{2} \theta}{d t^{2}}\right)+b_{b}\left(\frac{d \psi}{d t}+f \frac{d \theta}{d t}\right)+ \\
& +k_{b}(\psi+f \theta)=Q\left(r_{2}-r_{3}\right) \sin \omega t, \\
& J \frac{d^{2} \theta}{d t^{2}}+J_{b}\left(\frac{d^{2} \theta}{d t^{2}}+f \frac{d^{2} \psi}{d t^{2}}\right)+b_{b}\left(\frac{d \theta}{d t}+f \frac{d \psi}{d t}\right)+ \\
& +k_{b}(\theta+f \psi)=Q\left(r_{2}-r_{3}\right) \cos \omega t,
\end{aligned}
$$

где $x$-линейные перемещения вибрационного наконечника по центру тяжести колеблющейся системы в направлении координатной оси $X ; y$-линейные перемещения вибрационного наконечника по центру тяжести колеблющейся системы в направлении координатной оси $Y ; \psi-$ угловые перемещения вибрационного наконечника относительно координатной оси $Y$, проходящей через центр тяжести колеблющейся системы; $\theta$ - угловые перемещения вибрационного наконечника относительно координатной оси $X$, проходящей через центр тяжести колеблющейся системы; $b_{b}, c_{b}-$ значения приведенных коэффициентов неупругого и упругого сопротивлений бетонной смеси, как в направлении координатной оси $X$, так и в направлении координатной оси $Y$, определяемые в соответствии со способом вибрирования бетонной смеси: в полупространстве [8] или в форме [9]; $f$ - коэффициент трения бетонной смеси о поверхности вертикальных плит вибронаконечника; $J$ - момент инерции крестообразного глубинного уплотнителя относительно центра тяжести $C$ колеблющейся массы,

$$
J=J_{0}+m\left(r_{2}-r_{1}-r_{3}\right)^{2},
$$

где $J_{0}$ - момент инерции плоскостного глубинного уплотнителя относительно собственного центра тяжести $O_{2} ; J_{b}$ - момент инерции приведенной массы бетонной смеси относительно центра тяжести колеблющейся системы,

$$
J_{b}=m_{b} \frac{H^{2}}{12}+m_{b} r_{3}^{2} ;
$$

где $k_{b}, n_{b}$ - коэффициенты крутильной жесткости и неупругого сопротивления уплотняемой среды при угловых перемещениях колеблющейся системы относительно, как координатной оси $X$, так и координатной оси $Y$,

$$
k_{b}=k_{b 1}+k_{b 2} ; \quad n_{b}=n_{b 1}+n_{b 2},
$$

где $k_{b 1}, n_{b 1}-$ коэффициенты крутильной жесткости и неупругого сопротивления уплотняемой среды при угловых перемещениях колеблющейся системы относительно, как координатной оси $X$, так и координатной оси $Y$ на участке высотой $0,5 H-r_{3}$,

$$
\begin{aligned}
& k_{b 1}=\sum_{i=1}^{n_{1}} c_{b y} F_{i} z_{i}^{2} ; \\
& n_{b 1}=\sum_{i=1}^{n_{1}} b_{b y} F_{i} z_{i}^{2},
\end{aligned}
$$

где $k_{b 2}, n_{b 2}$ - коэффициенты крутильной жесткости и неупругого сопротивления уплотняемой среды при угловых перемещениях колеблющейся системы относительно, как координатной оси $X$, так и координатной оси $Y$ на участке высотой $0,5 H+r_{3}$,

$$
\begin{aligned}
& k_{b 2}=\sum_{i=1}^{n_{2}} c_{b y} F_{i} z_{i}^{2} ; \\
& n_{b 2}=\sum_{i=1}^{n_{2}} b_{b y} F_{i} z_{i}^{2},
\end{aligned}
$$

где $F_{i}$ - площадь равных элементарных участков, на которые разбита вертикальная плита по высоте, $F_{i}=F /\left(n_{1}+n_{2}\right) ; n_{1}, n_{2}$ - количество разбиений вертикальной плиты соответственно в верхней ее части на участке высотой $h$ и в нижней части на участке высотой $h_{1} ; Z_{i}$ - расстояние по вертикали от центра тяжести колеблющейся системы до $i$ того выделенного элемента; $c_{b y}, b_{b y}$ - значения удельных приведенных коэффициентов упругого и неупругого сопротивлений бетонной смеси, определяемые в соответствии со способом вибрирования бетонной смеси: в полупространстве [8] или в форме [9].

Для последовательного решения системы уравнений (2) и (3) произведем вычитание уравнения (3) из уравнения (2) и получим:

$$
\begin{gathered}
{\left[m+m_{b}(1-f)\right]\left(\frac{d^{2} x}{d t^{2}}-\frac{d^{2} y}{d t^{2}}\right)+b_{b}(1-f)\left(\frac{d x}{d t}-\frac{d y}{d t}\right)+} \\
+c_{b}(1-f)(x-y)=Q(\sin \omega t-\cos \omega t) .
\end{gathered}
$$

Произведем преобразование полученного выражения (13), принимая равным

$$
\zeta=x-y
$$

$$
Q(\sin \omega t-\cos \omega t)=\sqrt{2} Q \sin (\omega t-0,25 \pi)
$$

и получим уравнение колебаний в следующей форме

$$
\begin{gathered}
{\left[m+m_{b}(1-f)\right] \frac{d^{2} \zeta}{d t^{2}}+b_{b}(1-f) \frac{d \zeta}{d t}+c_{b}(1-f) \zeta=} \\
=\sqrt{2} Q(\sin \omega t-0,25 \pi) .
\end{gathered}
$$

На основании известных методов классической теории колебаний $[10,11]$, найдем решение уравнения (16) в следующем виде:

$$
\zeta(t)=A_{0} \sin \left(\omega t-\varphi_{1}\right),
$$


где $A_{0}$ - амплитуда колебаний; $\varphi_{1}-$ угол сдвига фаз между амплитудой возмущающей силы $Q$ и перемещением;

$$
\begin{gathered}
A_{0}=\sqrt{2} Q /\left\langle\left\{c_{b}(1-f)-\left[m+m_{b}(1-f)\right] \omega^{2}\right\}^{2}+\right. \\
\left.+\left[b_{b}(1-f) \omega\right]^{2}\right\rangle^{0,5} \\
\varphi_{1}=0,25 \pi+\varphi_{12} \\
\varphi_{12}=\operatorname{arctg} \frac{b_{b}(1-f) \omega}{c_{b}(1-f)-\left[m+m_{b}(1-f)\right] \omega^{2}} .
\end{gathered}
$$

Используя зависимости (14) и (17), найдем

$$
y=x-A_{0} \sin \left(\omega t-\varphi_{1}\right)
$$

Подставим выражение (21) в уравнение (2) и после преобразования получим новое уравнение, описывающее линейные колебания вибронаконечника по центру тяжести динамической системы в направлении координаты $X$ :

$$
\begin{aligned}
& {\left[m+m_{b}(1+f)\right] \frac{d^{2} x}{d t^{2}}+b_{b}(1+f) \frac{d x}{d t}+c_{b}(1+f) x=} \\
= & Q \sin \omega t+ \\
+ & A_{0} f \sqrt{\left(c_{b}-m_{b} \omega^{2}\right)^{2}+b_{b}^{2} \omega^{2}} \sin \left(\omega t-\varphi_{1}+\varphi_{2}\right),
\end{aligned}
$$

где

$$
\varphi_{2}=\operatorname{arctg} \frac{b_{b} \omega}{c_{b}-m_{b} \omega^{2}} .
$$

Решение уравнения (22) представим в следующем виде:

$$
x(t)=A_{11} \sin \left(\omega t-\varphi_{3}\right)+A_{12} \sin \left(\omega t-\varphi_{4}\right),
$$

где $A_{11}$ и $A_{12}$ - амплитуды колебаний; $\varphi_{3}$ и $\varphi_{4}$ - углы сдвига фаз между амплитудами возмущающих сил и амплитудами колебаний;

$$
\begin{gathered}
A_{11}=Q /\left\langle\left\{c_{b}(1+f)-\left[m+m_{b}(1+f)\right] \omega^{2}\right\}^{2}+\right. \\
\left.+\left[b_{b}(1+f) \omega\right]^{2}\right\rangle^{0,5} \\
A_{12}=A_{0} f \sqrt{\left(c_{b}-m_{b} \omega^{2}\right)+b_{b}^{2} \omega^{2}} /\left\langle\left\{ c_{b}(1+f)-\right.\right. \\
\left.\left.-\left[m+m_{b}(1+f)\right] \omega^{2}\right\}^{2}+\left[b_{b}(1+f) \omega\right]^{2}\right\rangle^{0,5} ; \\
\varphi_{3}=\operatorname{arctg} \frac{b_{b}(1+f) \omega}{c_{b}(1+f)-\left[m+m_{b}(1+f)\right] \omega^{2}} \\
\varphi_{4}=\varphi_{1}-\varphi_{2}+\varphi_{3} .
\end{gathered}
$$

Преобразовывая выражение (24), найдем в окончательном виде решение уравнения (22), описывающее линейные колебания вибронаконечника по центру тяжести динамической системы в направлении координатной оси $X$, т.е.

$$
x(t)=A_{1} \sin (\omega t-\varphi),
$$

где $A_{1}$ - амплитуда колебаний вибронаконечника по центру тяжести динамической системы в направлении координаты $X$,

$$
A_{1}=\sqrt{A_{11}^{2}+A_{12}^{2}+2 A_{11} A_{12} \cos \left(\varphi_{3}-\varphi_{4}\right)} ;
$$

где $\varphi$ - угол сдвига фаз между амплитудой возмущающих сил и амплитудой колебаний,

$$
\varphi=\operatorname{arctg} \frac{A_{11} \sin \varphi_{3}+A_{12} \sin \varphi_{4}}{A_{11} \cos \varphi_{3}+A_{12} \cos \varphi_{4}} .
$$

Для последовательного решения системы уравнений (4) и (5) вычтем из уравнения (4) уравнение (5). Тогда получим:

$$
\begin{gathered}
{\left[J+J_{b}(1-f)\right]\left(\frac{d^{2} \psi}{d t^{2}}-\frac{d^{2} \theta}{d t^{2}}\right)+n_{b}(1-f)\left(\frac{d \psi}{d t}-\frac{d \theta}{d t}\right)+} \\
+k_{b}(1-f)(\psi-\theta)=Q\left(r_{2}-r_{3}\right)(\sin \omega t-\cos \omega t) .
\end{gathered}
$$

Введя в уравнение (32) новую переменную

получим

$$
\gamma=\psi-\theta
$$

$$
\begin{aligned}
& {\left[J+J_{b}(1-f)\right] \frac{d^{2} \gamma}{d t^{2}}+n_{b}(1-f) \frac{d \gamma}{d t}+k_{b}(1-f) \gamma=} \\
& =Q\left(r_{2}-r_{3}\right)(\sin \omega t-\cos \omega t)= \\
& =\sqrt{2} Q\left(r_{2}-r_{3}\right)(\sin \omega t-0,25 \pi)
\end{aligned}
$$

Решение уравнения (34) можно представить в следующем виде:

$$
\gamma(t)=\Psi_{0} \sin \left(\omega t-\xi_{1}\right)
$$

где $\Psi_{0}$ - амплитуда угловых колебаний,

$$
\begin{aligned}
\Psi_{0}=\sqrt{2} Q\left(r_{2}-r_{3}\right) / & /\left\{k_{b}(1-f)-\left[J+J_{b}(1-f)\right] \omega^{2}\right\}^{2}+ \\
& \left.+\left[n_{b}(1-f) \omega\right]^{2}\right\rangle^{0,5} ;
\end{aligned}
$$

где $\xi_{1}$ - угол сдвига фаз между амплитудой момента возмущающей силы и угловым перемещением,

$$
\begin{gathered}
\xi_{1}=0,25 \pi+\xi_{12} ; \\
\xi_{12}=\operatorname{arctg} \frac{n_{b}(1-f) \omega}{k_{b}(1-f)-\left[J+J_{b}(1-f)\right] \omega^{2}} .
\end{gathered}
$$

Используя зависимости (33) и (35), найдем

$$
\theta=\psi-\Psi_{0} \sin \left(\omega t-\xi_{1}\right) .
$$

Подставим выражение (39) в уравнение (4) и после преобразования получим уравнение, описывающее угловые колебания вибронаконечника относительно координатной оси $Y$ :

$$
\begin{aligned}
& {\left[J+J_{b}(1+f)\right] \frac{d^{2} \psi}{d t^{2}}+n_{b}(1+f) \frac{d \psi}{d t}+k_{b}(1+f) \psi=} \\
& =Q\left(r_{2}-r_{3}\right) \sin \omega t+ \\
& +\Psi_{0} f \sqrt{\left(k_{b}-J_{b} \omega^{2}\right)^{2}+n_{b}^{2} \omega^{2}} \sin \left(\omega t-\xi_{1}+\xi_{2}\right),
\end{aligned}
$$


где

$$
\xi_{2}=\operatorname{arctg} \frac{n_{b} \omega}{k_{b}-J_{b} \omega^{2}} .
$$

Решение уравнения (40) представим в следующем виде:

$$
\psi(t)=\Psi_{11} \sin \left(\omega t-\xi_{3}\right)+\Psi_{12} \sin \left(\omega t-\xi_{4}\right),
$$

где $\Psi_{11}$ и $\Psi_{12}$ - амплитуды угловых колебаний; $\xi_{3}$ и $\xi_{4}-$ углы сдвига фаз между амплитудами моментов возмущающих сил и амплитудами угловых перемещений;

$$
\begin{gathered}
\Psi_{11}=Q\left(r_{2}-r_{3} /\left\langle\left\{k_{b}(1+f)-\left[J+J_{b}(1+f)\right] \omega^{2}\right\}^{2}+\right.\right. \\
\left.+\left[n_{b}(1+f) \omega\right]^{2}\right\rangle^{0,5} \\
\Psi_{12}=\Psi_{0} f \sqrt{\left(k_{b}-J_{b} \omega^{2}\right)+n_{b}^{2} \omega^{2}} /\left\langle\left\{ k_{b}(1+f)-\right.\right. \\
\left.\left.-\left[J+J_{b}(1+f)\right] \omega^{2}\right\}^{2}+\left[n_{b}(1+f) \omega\right]^{2}\right\rangle^{0,5} ; \\
\xi_{3}=\operatorname{arctg} \frac{n_{b}(1+f) \omega}{k_{b}(1+f)-\left[J+J_{b}(1+f)\right] \omega^{2}} \\
\xi_{4}=\xi_{1}-\xi_{2}+\xi_{3} .
\end{gathered}
$$

Преобразовывая выражение (42), найдем решение уравнения (40), описывающее угловые колебания вибронаконечника относительно координатной оси $Y$ в следующем окончательном виде:

$$
\psi(t)=\Psi_{1} \sin (\omega t-\xi),
$$

где $\Psi_{1}$ - амплитуда угловых колебаний вибронаконечника относительно координатной оси $Y$,

$$
\Psi_{1}=\sqrt{\Psi_{11}^{2}+\Psi_{12}^{2}+2 \Psi_{11} \Psi_{12} \cos \left(\xi_{3}-\xi_{4}\right)} ;
$$

где $\xi$ - угол сдвига фаз между амплитудой момента возмущающих сил и амплитудой угловых колебаний,

$$
\xi=\operatorname{arctg} \frac{\Psi_{11} \sin \xi_{3}+\Psi_{12} \sin \xi_{4}}{\Psi_{11} \cos \xi_{3}+\Psi_{12} \cos \xi_{4}}
$$

Закон движения рабочей поверхности вертикальной плиты, взаимодействующей $\mathrm{c}$ бетонной смесью в направлении координатной оси $X$, и вызывающей в этой бетонной среде нормальные напряжения, может быть на основании выражений (29) и (47) с учетом выражений (30), (31) и (48), (49) представлен в виде следующей функции:

$$
\begin{gathered}
X_{n}(z, t)=x(t)+z \psi(t) \quad \text { при } \\
-\left(0,5 H+r_{3}\right) \leq z \leq\left(0,5 H-r_{3}\right) .
\end{gathered}
$$

Подставляя в выражение (50) значения функций $x(t)(29)$ и $\psi(t)$ (47) получим зависимость для описания закона движения рабочей поверхности вертикальной плиты, контактирующей с бетонной смесью, в следующем виде:

$$
\begin{gathered}
X_{n}(z, t)=A_{x}(z) \sin \left[\omega t-\varphi_{x}(z)\right] \text { при } \\
-\left(0,5 H+r_{3}\right) \leq z \leq\left(0,5 H-r_{3}\right)
\end{gathered}
$$

где $A_{x}(z)$ - амплитуда перемещений вертикальной плиты, взаимодействующей рабочей поверхностью с бетонной смесью, в зависимости от координаты $z$,

$$
A_{x}(z)=\sqrt{A_{1}^{2}+\Psi_{1}^{2} z^{2}+2 A_{1} \Psi_{1} z \cos (\varphi-\xi)} ;
$$

где $\varphi_{x}(z)$ - угол сдвига фаз между амплитудой возмущающей нагрузки и амплитудой перемещения определенной точки на вертикальной плите с координатой $z$,

$$
\varphi_{x}(z)=\operatorname{arctg} \frac{A_{1} \sin \varphi+\Psi_{1} z \sin \xi}{A_{1} \cos \varphi+\Psi_{1} z \cos \xi} .
$$

Далее определим перемещение вибронаконечника в направлении координатной оси $Y$.

Из выражения (21) найдем значение функции

$$
x=y+A_{0} \sin \left(\omega t-\varphi_{1}\right)
$$

и, подставляя ее в зависимость (3), получим уравнение, описывающее линейные колебания вибронаконечника по центру тяжести динамической системы в направлении координаты $Y$ :

$$
\begin{gathered}
{\left[m+m_{b}(1+f)\right] \frac{d^{2} y}{d t^{2}}+b_{b}(1+f) \frac{d y}{d t}+c_{b}(1+f) y=} \\
=Q \cos \omega t- \\
-A_{0} f \sqrt{\left(c_{b}-m_{b} \omega^{2}\right)^{2}+b_{b}^{2} \omega^{2}} \sin \left(\omega t-\varphi_{1}+\varphi_{2}\right) .
\end{gathered}
$$

Решение уравнения (55) представим в следующем виде:

$$
y(t)=A_{11} \cos \left(\omega t-\varphi_{3}\right)-A_{12} \sin \left(\omega t-\varphi_{4}\right) .
$$

Здесь амплитуды колебаний $A_{11}$ и $A_{12}$ находятся из выражений (25) и (26), а углы $\varphi_{2}, \varphi_{3}$ и $\varphi_{4}$ определяются соответственно из выражений (23), (27) и (28).

Преобразовывая выражение (56), найдем окончательное решение уравнения (22), описывающее линейные колебания вибронаконечника по центру тяжести динамической системы в направлении координатной оси $Y$, т.е.

$$
y(t)=A_{2} \cos \left(\omega t-\varphi_{5}\right),
$$

где $A_{2}$ - амплитуда колебаний вибронаконечника по центру тяжести динамической системы в направлении координатой оси $Y$,

$$
A_{2}=\sqrt{A_{11}^{2}+A_{12}^{2}-2 A_{11} A_{12} \sin \left(\varphi_{3}-\varphi_{4}\right)}
$$

где $\varphi_{5}-$ угол сдвига фаз между амплитудой возмущающих сил и амплитудой колебаний,

$$
\varphi_{5}=\operatorname{arctg} \frac{A_{11} \sin \varphi_{3}-A_{12} \cos \varphi_{4}}{A_{11} \cos \varphi_{3}+A_{12} \sin \varphi_{4}} .
$$

Из зависимости (39) найдем

$$
\psi=\theta+\Psi_{0} \sin \left(\omega t-\xi_{1}\right) .
$$

Подставим выражение (60) в зависимость (5) и после преобразования получим уравнение, описывающее угловые колебания вибронаконечника относительно координатной оси $X$ : 


$$
\begin{aligned}
& {\left[J+J_{b}(1+f)\right] \frac{d^{2} \theta}{d t^{2}}+n_{b}(1+f) \frac{d \theta}{d t}+k_{b}(1+f) \theta=} \\
& =Q\left(r_{2}-r_{3}\right) \cos \omega t- \\
& -\Psi_{0} f \sqrt{\left(k_{b}-J_{b} \omega^{2}\right)^{2}+n_{b}^{2} \omega^{2}} \sin \left(\omega t-\xi_{1}+\xi_{2}\right) .
\end{aligned}
$$

Здесь амплитуды угловых колебаний $\Psi_{11}$ и $\Psi_{12}$ находятся из выражений (43) и (44), а углы $\xi_{2}$, $\xi_{3}$ и $\xi_{4}$ определяются соответственно из выражений (41), (45) и (46).

Преобразовывая выражение (77), найдем решение уравнения (20), описывающее угловые колебания вибронаконечника относительно координатной оси $X$ в следующем окончательном виде:

$$
\theta(t)=\Psi_{2} \cos \left(\omega t-\xi_{5}\right),
$$

где $\Psi_{2}$ - амплитуда угловых колебаний вибронаконечника относительно координатной оси $X$,

$$
\Psi_{2}=\sqrt{\Psi_{11}^{2}+\Psi_{12}^{2}-2 \Psi_{11} \Psi_{12} \sin \left(\xi_{3}-\xi_{4}\right)} ;
$$

где $\xi_{5}-$ угол сдвига фаз между амплитудой момента возмущающих сил и амплитудой угловых колебаний,

$$
\xi_{5}=\operatorname{arctg} \frac{\Psi_{11} \sin \xi_{3}-\Psi_{12} \cos \xi_{4}}{\Psi_{11} \cos \xi_{3}+\Psi_{12} \sin \xi_{4}} .
$$

Закон движения рабочей поверхности вертикальной плиты, взаимодействующей с бетонной смесью в направлении координатной оси $Y$, и вызывающей в этой бетонной среде нормальные напряжения, может быть на основании выражений (57) и (62) представлен в виде следующей функции:

$$
\begin{gathered}
Y_{n}(z, t)=y(t)+z \theta(t) \quad \text { при } \\
-\left(0,5 H+r_{3}\right) \leq z \leq\left(0,5 H-r_{3}\right) .
\end{gathered}
$$

Подставляя в выражение (65) значения функций $y(t)(57)$ и $\theta(t)(62)$ получим зависимость для описания закона движения рабочей поверхности вертикальной плиты, контактирующей с бетонной смесью, в следующем виде:

$$
\begin{gathered}
Y_{n}(z, t)=A_{y}(z) \sin \left[\omega t+\varphi_{y}(z)\right] \text { при } \\
-\left(0,5 H+r_{3}\right) \leq z \leq\left(0,5 H-r_{3}\right),
\end{gathered}
$$

где $A_{y}(z)$ - амплитуда перемещений вертикальной плиты, взаимодействующей рабочей поверхностью с бетонной смесью, в зависимости от координаты $z$,

$$
A_{y}(z)=\sqrt{A_{2}^{2}+\Psi_{2}^{2} z^{2}+2 A_{2} \Psi_{2} z \cos \left(\varphi_{5}-\xi_{5}\right)} ;
$$

где $\varphi_{y}(z)$ - угол сдвига фаз между амплитудой возмущающей нагрузки и амплитудой перемещения определенной точки на вертикальной плите с координатой $z$,

$$
\varphi_{y}(z)=\operatorname{arctg} \frac{A_{2} \sin \varphi_{5}+\Psi_{2} z \sin \xi_{5}}{A_{2} \cos \varphi_{5}+\Psi_{2} z \cos \xi_{5}} .
$$

Анализ выражений $(51,52)$ и $(66,67)$ показывает, что крестообразный наконечник предлагаемого глубинного виброуплотнителя совершает при работе пространственные колебания в горизонтальной плоскости во двух взаимно перпендикулярных направлениях, захватывая и обеспечивая большие объемы вибрационной обработки. При этом благодаря переменному амплитудно-частотному вибрационному воздействию обеспечивается эффективное уплотнение бетонной смеси. Такое вибрационное воздействие вызывает в уплотняемой среде нормальное напряженно-деформированное состояние с изменяющимися параметрами в горизонтальной плоскости, которое является определяющим в разрушении структурных связей, переводу бетонной смеси в тиксотропное состояние. При этом резко уменьшаются силы внутреннего трения в смеси за счет выделения в межзерновое пространство свободной воды, играющей роль смазки, происходит интенсификация процесса уплотнения за счет вытеснения из уплотняемой смеси воздуха, заполнения вяжущим микротрещин на поверхности минеральных частиц, их переориентации и сближения с образованием более плотной упаковки.

ВЫВОДЫ. Составлена расчетная схема принципиально новой конструкция крестообразного глубинного уплотнителя, который состоит из погружаемого в бетонную смесь уплотняющего наконечника, в верхней части которого смонтирован вибровозбудитель круговых колебаний. Определены физикомеханические характеристики бетонной смеси, взаимодействующей с глубинным уплотнителем. Составлена система уравнений для описания прямолинейных и крутильных колебаний крестообразного уплотнителя. Установлена закономерность движения рабочих поверхностей крестообразного глубинного уплотнителя, взаимодействующих с уплотняемой бетонной смесью в рабочем режиме. Проведенными исследованиями установлены основные параметры вибрационного воздействия на уплотняемую среду в виде переменного амплитудно-частотного деформирования уплотняемой среды. Предлагаемый крестообразный глубинный уплотнитель может найти широкое применение в практике строительства.

\section{ЛИТЕРАТУРА}

1. Juradin S., Baloević G., Harapin A. Impact of Vibrations on the Final Characteristics of Normal and Self-compacting Concrete. Journal of Materials Research. 2014, Vol. 17(1), pp. 178-185.

2. Sudarshan N. M., Chandrashekar R. T. Vibration Impact on Fresh Concrete of Conventional and UHPFRC. International Journal of Applied Engineering Research. 2017, Vol. 12, 8thedn, pp. 1683-1690.

3. Koh H. B., Yeoh D., Shahidan S. Effect of revibration on the compressive strength and surface hardness of concrete. IOP Conf. Series: Materials Science and Engineering. 2017, Vol. 271, 012057, pp. 1-6.

4. Gutierrez J., Ruiz E., Trochu F. (2013), Highfrequency vibrations on the compaction of dry fibrous reinforcements. Journal of Advanced Composite Materials. 2013, Vol. 22 (1), pp. 13-27 
5. Волков С. А., Евтюков С. А. Строительные машины. СПб.: ДНК, 2012. 597 с.

6. Стаценко А. С. Технология каменных работ в строительстве. Минск: Выш. шк. 2010. 255 с.

7. Жанар Батсайхан. Разработка крестообразного глубинного уплотнителя бетонных смесей. Вісник Кре-менчуиького національного університету імені Михайла Остроградського. 2018. Вип. 6 (113). C. 58-63.

8. Maslov A., Janar Batsaikhan, Puzyr R., SalenkoYu. The Determination of the Parameters of a Vibra- tion Machinef the Internal Compaction of Concrete Mixtures. International Journal of Engineering \& Technology, 2018, Vol. 7 (4.3), pp 12-19.

9. Maslov O., Janar Batsaikhan, SalenkoYu. The Theory of Concrete Mixture Vibratory Compacting. International Journal of Engineering \& Technology, 2018, Vol. 7 (3.2), pp 239-244.

10. Маслов А. Г. Иткин А. Ф., Саленко Ю. С. Вибрационные машины для приготовления и уплотнения бетонных смесей: монография. Кременчуг: ЧП Щербатых А.В, 2014. 324 с.

\section{THEORETICAL STUDY OF CRUCIFORM DEEP SEALER CONCRETE MIXTURES}

\section{A. Maslov, Janar Batsaikhan}

Kremenchuk Mykhailo Ostrohradskyi National University

vul. Pershotravneva, 20, Kremenchuk, 39600, Ukraine. E-mail: kmto.43@gmail.com

Purpose. The article deals with theoretical definition of the laws of motion and rational parameters of the crossshaped deep vibration seal of concrete mixtures of different consistency. Methodology. The design scheme of a crossshaped deep seal interacting with a concrete medium, physical and mechanical properties of which are presented in the form of elastic, dissipative and inertial forces, is presented. A fundamentally new design of the cross-shaped deep seal, which consists of a sealing tip immersed in a concrete mixture, is proposed. The tip is made in the form of crossconnected vertical plates. On top of the sealing of the cross-shaped tip with the help of the support ring is mounted a vibration exciter circular oscillation. The fundamentals of the operating principle of the method of compaction of the concrete mixture by variable amplitude-frequency deformation of the concrete mixture, which occurs as a result of spatial oscillations of the proposed cross-shaped deep seal, are shown. Results. A system of equations describing the rectilinear oscillations of the center of gravity of the dynamic system under study in the horizontal plane in mutually perpendicular directions, as well as torsional oscillations with respect to the coordinate axes passing through the center of gravity of the cruciform deep seal is developed. The moments of inertia of the oscillating dynamic system are determined taking into account the physical and mechanical characteristics of the compacted medium, the coefficients of torsional stiffness and inelastic resistance of the concrete mixture are found. Originality. The regularity of the movement of the working surfaces of the cross-shaped sealing tip in the horizontal plane in mutually perpendicular directions and causing in the concrete mixture variable amplitude-frequency vibration effect, which provides in the compacted medium normal stress-strain state with varying parameters in the horizontal plane, which is decisive in the destruction of structural bonds, the transfer of the concrete mixture to the thixotropic state. Practical. The obtained results allow: to justify the rational parameters of the cruciform deep seal, performing spatial vibrations and causing variable amplitudefrequency deformation in the concrete mixture, contributing to effective compaction; to find rational modes of vibration impact on concrete mixtures of different consistency. The proposed cross-shaped deep seal can be widely used in construction practice. References 10 , tables 0 , figures 1 .

Key words: planar deep seal, concrete mix, vibration seal.

\section{REFERENCES}

1. Juradin, S., Baloević, G., Harapin, A. (2014), Impact of Vibrations on the Final Characteristics of Normal and Self-compacting Concrete, Journal of Materials Research, Vol. 17(1), pp. 178-185.

2. Sudarshan, N. M., Chandrashekar, R. T. (2017), Vibration Impact on Fresh Concrete of Conventional and UHPFRC, International Journal of Applied Engineering Research, Vol. 12, $8^{\text {th }}$ edn, pp. 1683-1690.

3. Koh, H. B., Yeoh, D., Shahidan, S. (2017), Effect of re-vibration on the compressive strength and surface hardness of concrete, IOP Conf. Series: Materials Science and Engineering, Vol 271, 012057, pp. 1 - 6.

4. Gutierrez, J., Ruiz, E., Trochu, F. (2013), Highfrequency vibrations on the compaction of dry fibrous reinforcements, Journal of Advanced Composite Materials, Vol. 22 (1), pp. $13-27$.

5. Volkov, S. A., Evtyukov, S. A. (2012), Stroitel'nye mashiny [Construction machinery]. "DNK", $\mathrm{SPb}$, Russia.
6. Stacenko, A. S. (2010) Tekhnologiya kamennyh rabot $v$ stroitel'stve [Technology of stone works in construction], "Vysh. shk.", Minsk, Belorussiya.

7. Batsaikhan, J. (2018), Development of crossshaped deep seal of concrete mixtures, Transactions of Kremenchuk Mykhailo Ostrohradskyi National University, Kremenchug: KRNU, Issue (113), pp. 58 - 63.

8. Maslov, A., Batsaikhan, J., Puzyr, R, Salenko, Yu. (2018), The Determination of the Parameters of a Vibration Machinef the Internal Compaction of Concrete Mixtures, International Journal of Engineering \& Technology, Vol. 7 (4.3), pp 12-19.

9. Maslov, O., Batsaikhan, J., Salenko, Yu. (2018), The Theory of Concrete Mixture Vibratory Compacting, International Journal of Engineering \& Technology, Vol. 7 (3.2), pp 239-244.

10. Maslov, A. G., Itkin, A. F, Salenko, Y. S. (2014), Vibratsionnyie mashinyi dlya prigotovleniya $i$ uplotneniya betonnyih smesey [Vibrating machines for the preparation and compaction of concrete mixes], PP Cherbatyh, Kremenchuk, Ukraine. 